\title{
AMENDMENTS
}

\section{Author Correction: Regional variation limits applications of healthy gut microbiome reference ranges and disease models}

Yan He, Wei Wu, Hui-Min Zheng, Pan Li, Daniel McDonald, Hua-Fang Sheng, Mu-Xuan Chen, Zi-Hui Chen, Gui-Yuan Ji, Zhong-Dai-Xi Zheng, Prabhakar Mujagond, Xiao-Jiao Chen, Zu-Hua Rong, Peng Chen, Li-Yi Lyu, Xian Wang, Chong-Bin Wu, Nan Yu, Yan-Jun Xu, Jia Yin, Jeroen Raes, Rob Knight ID, Wen-Jun Ma and Hong-Wei Zhou $\mathbb{D}^{\mathrm{D}}$

Correction to: Nature Medicine https://doi.org/10.1038/s41591-018-0164-x, published online 27 August 2018.

In the version of this article originally published, in the sentence "Applying the same approach to obesity (Fig. 2b), MetS (Fig. 2c) and fatty liver (Fig. 2d) yielded similar results," two figure panels were cited incorrectly. The data for obesity are in Fig. 2c, and the data for MetS are in Fig. 2b. The sentence has been updated with the correct citations in the print, PDF and HTML versions of the article.

Published online: 24 September 2018

https://doi.org/10.1038/s41591-018-0219-z

\section{Author Correction: MitoTALEN reduces mutant mtDNA load and restores tRNA ${ }^{\text {Ala }}$ levels in a mouse model of heteroplasmic mtDNA mutation}

Sandra R. Bacman, Johanna H. K. Kauppila, Claudia V. Pereira, Nadee Nissanka, Maria Miranda (D), Milena Pinto (D), Sion L. Williams, Nils-Göran Larsson (D), James B. Stewart (D) and Carlos T. Moraes

Correction to: Nature Medicine https://doi.org/10.1038/s41591-018-0166-8, published online 24 September 2018.

In the version of this article originally published, there was an error in Fig. 1a. The m.5024C>T mutation, shown as a green $\mathrm{T}$, was displaced by one base. The error has been corrected in the print, HTML and PDF versions of this article.
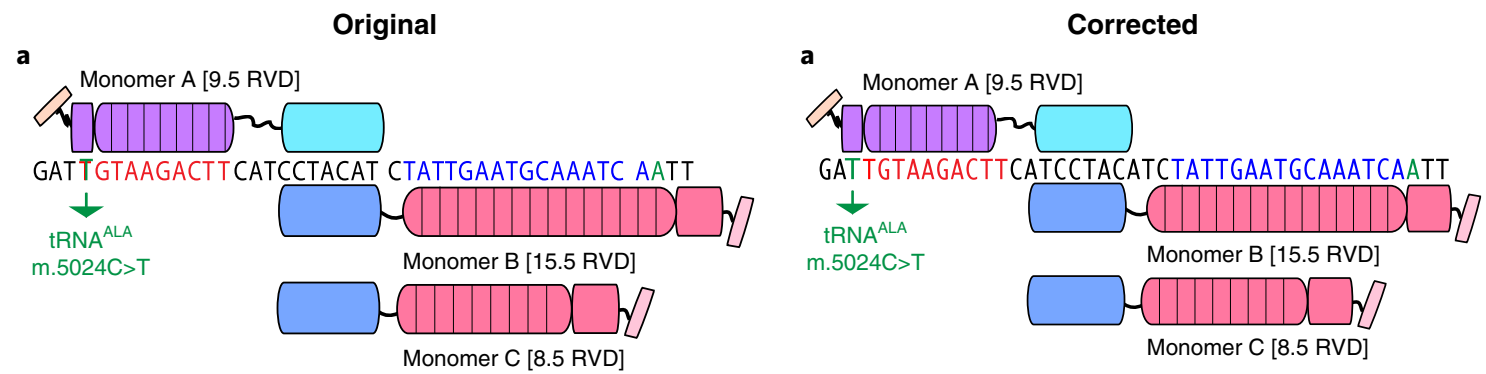

Fig. 1 | Original and Corrected.

Published online: 5 October 2018

https://doi.org/10.1038/s41591-018-0234-0 